\title{
Comparison of Religious Cognitive Behavioral Therapy, Cognitive Behavioral Therapy, and Citalopram on Depression and Anxiety among Women with Breast Cancer: A Study Protocol for a Randomized Controlled Trial
}

\author{
Seyed Hamzeh Hosseini ${ }^{1,2}$, Alireza Rafiei ${ }^{3}$, Ghasem Janbabai ${ }^{4,5}$, Abdolhakim Tirgari ${ }^{6}$, \\ Aliasghar Zakavi ${ }^{7}$, Jamshid Yazdani ${ }^{8}$, Jafar Bolhari ${ }^{9}$, Manhood Golzari ${ }^{10}$, \\ Nazanin Vaezzadeh ${ }^{1,11}$ and Zahra Esmaeili Douki ${ }^{1,11^{*}}$ \\ 'Psychiatry and Behavioral Sciences Research Center, Addiction Institute, Mazandaran University of Medical \\ Sciences, Sari, Iran \\ 2Department of Psychosomatic, Imam Khomeini Hospital, Mazandaran University of Medical Sciences, Sari, Iran \\ ${ }^{3}$ Molecular and Cell Biology Research Center, Faculty of Medicine, Mazandaran University of Medical Sciences, \\ Sari, Iran \\ ${ }^{4}$ Department of Internal Medicine, Mazandaran University of Medical Sciences, Sari, Iran \\ ${ }^{5}$ Department of Hematology and Oncology, Mazandaran University of Medical Sciences, Sari, Iran \\ ${ }^{6}$ Department of Psychiatry Member of Psychiatry, Behavioral Sciences Research Center, Sari, Iran \\ ${ }^{7}$ Department of Islamic Thought, Faculty of Medicine, Mazandaran University of Medical Science, Sari, Iran \\ ${ }^{8}$ Department of Biostatistics, Health Sciences Research Center, Faculty of Health, \\ Mazandaran University of Medical Sciences, Sari, Iran \\ ${ }^{9}$ Mental Health Research Center (MHRC), Tehran Psychiatric Institute, Iran University of Medical Sciences (IUMS), \\ Tehran, Iran \\ ${ }^{10}$ Faculty of Psychology and Educational Sciences, Allame Tabatabai University, Tehran, Iran \\ ${ }^{11}$ Department of Pediatric Nursing, Faculty of Nursing and Midwifery, Mazandaran University of Medical Sciences, \\ Sari, Iran;zesmaeilidouki@yahoo.com
}

\begin{abstract}
There has been evidence supporting the reduction of depression and anxiety by religious psychotherapy in cancer patients, however, there have been scarce randomized controlled trials. Therefore, there is a need for replication in a well-designed study to investigate the efficacy of these interventions among depressed women with breast cancer. A randomized controlled trial is designed to be conducted on 160 women with breast cancer. Participants will be screened for anxiety and depression diagnosed by clinical interview based on the DSM-IV criteria and the Hospital Anxiety Depression Scale. Golriz and Baraheni's Religious Attitude Questionnaire will be used to assess the religious attitude. Individuals meeting the prerequisites will be randomly allocated to four groups, each containing 40 participants, including three intervention groups (RCBT, CBT, and citalopram) and one control group (usual breast cancer treatment). An instrument by the EORTCQLQ-C30 and BR-23 will be used to measure the quality of life. Blood tests will be taken to assess biomarkers with the (ELISA) method. The results will determine whether RCBT is more effective than other treatments. If so, the outcomes of the study will have implications not only for the management of similar problems in cancer patients but also for the management of other chronic diseases.
\end{abstract}

${ }^{*}$ Author for correspondence 
Keywords: Anxiety, Biomarker, Breast Cancer, Citalopram, Cognitive Behavioral Therapy, Depression, Quality of Life, Religious Cognitive Behavioral Therapy

\section{Background}

Breast cancer is one of the most common malignancies among women worldwide ${ }^{1,2}$. It has been observed that diagnosis, surgery, chemotherapy, and radiotherapy may contribute to psychological symptoms ${ }^{3,4}$, cognitive malfunction $^{5-7}$, and changes in daily life ${ }^{8,9}$. Recently, researchers have emphasized the relationship between psycho-neuro-immunological factors and depression ${ }^{10,11}$. Several studies have shown significant increases in the production ofpro-inflammatorycytokines (IL-6,TNF- $\alpha)^{12}$, and (IFN- $\gamma$ ) among depressed patients ${ }^{12,13}$, although anti-inflammatory cytokines such as IL-10modulate depressive-like behavior ${ }^{14,15}$. However, the results of other studies have shown no association between depression and cytokines ${ }^{16,17}$. There are several effective treatments for anxiety, depression and normalization of cytokines. From the psychiatric point of view, pharmacological therapies have been considered as first-line treatments for mental disorders ${ }^{18}$. As a second-generation antidepressant drug, citalopram has recently been extensively used in the treatment of depression symptoms ${ }^{19}$. Decreases in the anxiety symptoms along with those of depression and a lack of interaction with tamoxifen, which prevents breast cancer relapse, are the other advantages of this drug ${ }^{20}$. However, drug interventions and their side effects may cause problems for most patients even when prescribing serotonin selective reuptake inhibitor (SSRI) as safety drugs $^{21}$. Instead, psycho-spiritual therapy as a nonpharmacological intervention has proven to be a useful treatment modality ${ }^{22,23}$. There has been evidence to support decreases in psychological problems ${ }^{24,25}$, reductions in adverse biological changes ${ }^{26-28}$ and improvements in the quality of life using religious intervention in cancer patients ${ }^{29-31}$, particularly when the interventions are based on a cognitive-behavioral structure ${ }^{29,32,33}$. Nonetheless, few randomized controlled trials in religious therapy have been conducted ${ }^{29,34}$ and there are conflicting results $^{35,36}$. Furthermore, some studies have reported serious limitations in methodology $y^{9,29,37,38}$. Accordingly, there is a need replication in a more definitive and welldesigned study to evaluate the effects of RCBT, CBT, and citalopram, on anxiety, depression, and biomarkers levels in women with breast cancer. This article includes the protocol of the study, while the findings will be presented when the study is accomplished.

\section{Objectives and Hypotheses}

The aim of this study is twofold. First, the study compares the effects of RCBT, CBT, and citalopram on anxiety and depression, in women with breast cancer. The secondary aims will be to compare the effects of RCBT, CBT, and citalopram on the quality of life, and biomarkers levels of IL6, IL10, and TNF- $\alpha$, IFN- $\gamma$, DHEA, and cortisol, in women with breast cancer.

It is hypothesized that RCBT will be more effective than CBT and citalopram on anxiety, depression, quality of life and biomarkers levels of IL6, IL10, TNF- $\alpha$, IFN- $\gamma$, DHEA and cortisol in women with breast cancer.

\section{Outcome}

The primary outcome will be reductions in the anxiety and depression scores immediately, and at 6, 9, and 12 months after the intervention. The secondary outcomes are increases in the quality of life scores immediately and at 6,9 , and 12 months after the interventions, and normalizing biomarkers levels immediately and 12 months after the intervention.

\section{Methods and Design}

This study was approved by the Behavioral Sciences Research Center in Mazandaran University of Medical Science (2013-12-04), and we have received an ethical consensus from the Ethics Committee in the Research Deputy of Mazandaran University of Medical Sciences (92193) that is fully compliant with the Helsinki Declaration of 2008. This randomized controlled trial with parallel groups is supposed to evaluate the effect of RCBT compared to CBT, citalopram, and usual breast cancer treatment on the reduction of anxiety and depression in women with breast cancer in Tuba Clinic in Sari. This center was located in Northern Iran. The participants will be randomly allocated to one of three intervention groups (RCBT, CBT, or citalopram) and one control group (usual breast cancer treatment). Participants will have been referred to this center for follow-up treatment from throughout the province. 


\subsection{Inclusion Criteria}

1) Women with breast cancer who are being treated in Tuba Clinic. 2) Age of less than 65 years. 3) Being at a clinical level of 1, 2 or 3 for breast cancer. 4) Having undergone surgery, chemotherapy, or radiotherapy. 5) Ability to understand and communicate in Farsi. 6) Education level of junior middle school or higher. 7) Meeting the DSMIV criteria for anxiety and depression ${ }^{39} .8$ ) Obtaining a score of $\geq 8$ for depression and anxiety based in HADS (Iranian version $)^{40}$. 9) Providing written consent for study participation and 10) Expressing religious ${ }^{41}$.

\subsection{Exclusion Criteria}

1) Participants with known psychiatric history such as schizophrenia, bipolar disorder, and serious suicidal thoughts based on the clinical interview (DSM-IV) performed by a psychiatrist. 2) Participants with a history of physical illnesses or any kind of malignancy preventing them from attending the treatment sessions. 3) Participants who have taken part in RCBT or CBT or other psychotherapy courses, and 4) Patients taking citalopram for the previous 2 weeks.

\section{Procedure}

The researcher will first assess archived records related to women with breast cancer referred to Tuba Clinic. After applying the inclusion and exclusion criteria, patients who meet the required conditions will be selected. Then, the researcher will contact the patients and briefly explain the study. If patient agree to participate in the study (oral consent), they will be invited to participate at Tuba Clinic at the appointed time. All participants will be asked if they are religious people (yes or no); for those who answered yes, their religious attitude intensity will be determined by using Golriz and Baraheni's religious attitude questionnaire (Iranian version) ${ }^{42}$. If their score is above 25, they will be enrolled in this trial. At the baseline assessment, inclusion and exclusion criteria will be checked again and participants will be informed about the details of the research project by the researcher. After obtaining the written consent, the researcher will give them the HADS questionnaire to assess anxiety and depression symptoms. Participants with HADS scores of 8 and more will be referred to a psychiatrist for a clinical interview based on the DSM-IV. Participants with mental disorders such as schizophrenia, bipolar disorder, and serious thoughts of suicide will be excluded and referred to treatment. Thereafter, to assess quality of life, the researcher will use a questionnaire by the European Organization for Research and Treatment of Cancer Quality of Life (EORTCQLQ-C30) and a breast cancer specific questionnaire (BR-23) ${ }^{43}$. Blood tests will be taken to assess biomarker levels of IL6, IL10, TNF- $\alpha$, IFN- $\gamma$, DHEA, and cortisol with ELISA method. Eligible participants will be randomly allocated to the intervention or control groups. The therapists will have completed training courses related to RCBT and CBT interventions. Then, the intervention will be performed by therapists. Assessments will be conducted at baseline, immediately after intervention, and at 6,9, and 12 months after the intervention. Participants will be called once every 2 weeks throughout the study, so that their problems and probable questions can be addressed. Table 1 shows the different assessment times of the questionnaires. Figure 1 contains the expected participant flow chart.

Table 1. Measurement of outcome variables, at baseline (pre intervention), immediately after intervention, 6, 9 and 12 months after intervention

\begin{tabular}{|c|c|c|c|c|c|c|}
\hline Variable & Measurement tool & Time of a & sessment & & & \\
\hline & & Baseline & after intervention & 6 & 9 & 12 month after intervention \\
\hline Depression and anxiety & Hospital anxiety and depression & * & * & * & * & * \\
\hline Clinical interview & DSM -IV & * & & & & \\
\hline Religious attitude & $\begin{array}{l}\text { Golriz and Baraheni's religious } \\
\text { attitude }\end{array}$ & * & & & & \\
\hline Quality of life & EORTCQLQ-C30 \& BR-23 & * & * & * & * & * \\
\hline Blood test Biomarkers & ELISA & & & & & \\
\hline $\begin{array}{l}\text { (IL6, IL10, TNF- } \alpha, \text { IFN } \gamma \text {, } \\
\text { DHEA, cortisol) }\end{array}$ & & * & & & & * \\
\hline
\end{tabular}




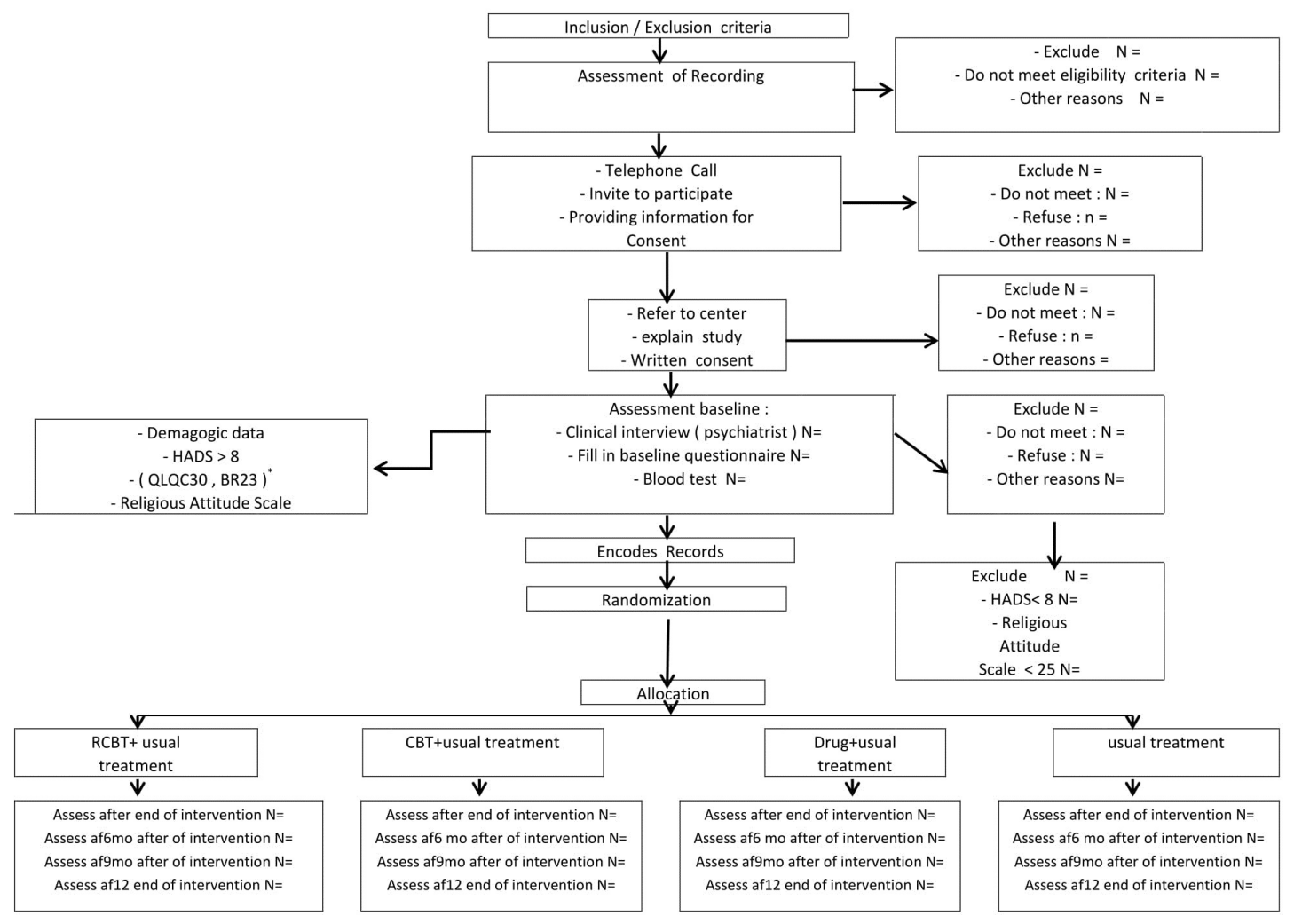

Figure 1. Flowchart of study design.

\subsection{Randomization}

Eligible participants are selected based on random numbers from the table and then randomly allocated into four groups of 40. Participants are randomized using block randomization to ensure equal group sizes. Randomization will be stratified by age $(\leq 48, \geq 48)$ and level of education ( $\leq$ high middle school, and $\geq$ high middle school). The participants will be informed about the treatments and control groups after randomization.

\subsection{Sampling}

Participants will be recruited from Tuba Clinic and, to account for any attrition that may occur, multiple imputations will be used. Furthermore, by increasing the number of the participants up to $25 \%$, to the study will lessen the attrition rate. According to previous studies ${ }^{44}$ with $\alpha=5 \%$ and a power analysis of $80 \%$, the number of participants needed has been determined to be 160 patients, with 40 in each group.

\subsection{Instrumentation}

Structured clinical interview (DSM-IV): A psychiatrist will conduct a structured clinical interview based on the DSM-IV. If the patient has any kind of mental disorders (bipolar, schizophrenia, serious suicidal thoughts) diagnosed, or the patient has a history of taking psychiatric drugs, we will exclude him/her from the study and refer him/her to a psychiatrist to be treated. Hospital Anxiety Depression Scale (HADS): This scale contains 14 questions including two parts measuring depression and anxiety. The scale has four options and each answer is scored from 0 to 3 .The validity and reliability of this scale in Iran has been confirmed. This instrument has been found reliable with a Cranach's index of 0.78 for anxiety and 0.86 for depression ${ }^{40}$.The European Organization for Research and Treatment of Cancer Quality of Life Group (EORTC QLG) will be used to measure quality of life in the patients ${ }^{43}$. The another questionnaire used is the breast cancer specific questionnaire (BR-23) ${ }^{45}$. These two questionnaires were translated and validated in Persian ${ }^{46}$. Gloria and Baraheni's Religious Attitude Questionnaire: This religious Iranian questionnaire contains 25 questions in a five-point Likert-scale from zero to four (the maximum total score is 100$)^{47}$. 


\subsection{Blood Test}

To measure the biomarkers of IL6, IL10, DHEA, cortisol, TNF- $\alpha$ and IFN- $\gamma$, blood tests will be obtained by a laboratory expert (research assistant) in the laboratory of Tuba Clinic in Sari. It will be conducted before, immediately after the intervention. From each participant, 5-7 cc peripheral blood in the form of a clot will be obtained; the serums will be immediately extracted and divided into small amounts to be transmitted to the lab of the cellular and molecular biology research center. Then, it will be stored in cold chain compliance in $-80^{\circ} \mathrm{C}$ up to the experiment date. All stages of transmission and processing will be identical for all samples in all four groups. Cytokines of TFN- $\alpha$, IFN- $\gamma$, IL-6, and IL-10 will be tested using the sandwich ELISA method.

\subsection{Interventions}

After randomization, the RCBT and CBT groups will receive 12 one-hour weekly sessions of an intervention along with their usual breast cancer treatment. The drug intervention group will receive citalopram $20-40$ mg every evening based on a physician's order accompanied by usual breast cancer treatment for 3 months. The control group will receive only usual breast cancer treatment. During the interventions and after that, the research assistant will call the participants in all of the groups, every 2 weeks to monitor and ensure their well-being. If the symptoms worsen, they will be excluded from the study and will be referred to psychiatrists for further treatment.

\subsection{Cognitive Behavioral Therapy}

Cognitive behavioral therapy is an interactional approach that helps participants to develop treatment strategies via the dynamic modification of cognition and behavior. According to the protocol and treatment techniques developed by Beck ${ }^{48}$, the topics of each session will already be determined. To meet the specific needs of the patients with breast cancer, the techniques developed by the Becks will be modified.

\subsection{Religious Cognitive Behavioral Intervention}

A researcher-made RCBT manual will be devised with the structure and content adapted from Judith Beck's cognitive-behavior model. RCBT teaches patients to use their own religious teachings, doctrines, and behaviors to help change the maladaptive beliefs, values, and behaviors ${ }^{20}$. This model will be modified to be applicable for the interventions related to religious. Religious concepts such as faith and trust, prayer, sacred, relationship with God, by searching the religious books, literature and electronic sources under the supervision of experts will be collected. In this way, Iranian sociocultural backgrounds will be considered.

\subsection{Pharmacologic Intervention}

Citalopram 20-40 mg will be administered to this group every evening based on a psychiatrist's order for 3 months in addition to the usual breast cancer treatment. During this period, they will be visited every 2 weeks and will be questioned about the concomitant use of medications and symptoms, side effects, and illnesses. This way, if participants display adverse effects, they will be excluded from the study and refer to a psychiatrist for follow-up treatment. Citalopram will be provided from Sobhan Pharmaceutical Company in Iran in the form of oral tablets in 20 and $40 \mathrm{mg}$ dosages; it has been officially licensed by Iran Health Ministry.

\section{Usual Breast Cancer Treatment}

The control group will solely receive the usual breast cancer treatment, which refers to physical care, medication, nutrition, activities, routine psychological care, and periodic visits for follow-up. An instructional pamphlet containing this information will be given to the patients. Similar to other three groups, the research assistant will monitor this group once every 2 weeks via telephone. If the symptoms worsen, they will be excluded from the study and will be referred to psychiatrists for further treatment.

\section{Data Analysis}

The data will be analyzed with SPSS for Windows, version 20. Descriptive statistics (mean frequency and percentage) and analytical statistics (t-test, chi-square, ANOVA, log rank test, Cox regression, Kaplan-Meier, and survival analysis) will be employed to analyze the collected data. ANCOVA and regression will also be employed for stratifying data by age and levels of education. 


\section{Discussion}

This study will be conducted to investigate the effects of RCBT compared to CBT and citalopram therapy on the depression and anxiety of women suffering from breast cancer. The findings of this study will indicate whether RCBT can work more efficiently than other interventions in the treatment of depression, anxiety, and physiological changes caused by these comorbidities. If so, the outcomes of the study will have implications not only for religious psychological management in breast cancer but also for the management of similar problems in other chronic diseases. This article includes the protocol of the study, and findings will be presented when the study is accomplished.

\section{Safety Aspects and Medical Complications}

Attention should be paid to safety parameters including the diagnosis of mental disorders and serious problems throughout the treatment and 1-year follow-up.

Recording the adverse effects that may occur during the intervention and the following 12 months, as they may lead to death, life-threatening issues, a need for hospitalization, and everything leading to resistance to treatment and inability in the patient.

Recording every type of adverse effects diagnosed during the study according to clinical interviews by the psychiatrist and coding in the report forms.

Every diagnosed adverse effect should be reported to the Ethics Committee.

\section{Ethics}

The participants will be informed about the purpose of the study and will be assured about the safety of the interventions. They will be also made aware of the confidentiality of their personal information. Permission for doing the project came from Ethics Committee of Research Deputy of Mazandaran University of Medical Sciences and from Tuba Specialist Clinic. Every question posed by the participants will be answered by the researcher. Participation is voluntary and participants will be informed that they can withdraw their consent to participate at any time. Written consent will be obtained from all the participants. Because the participants get tired quickly, all interviews will be brief. The RCBT and CBT therapists have passed the relevant courses, have the experience in this regard, and can identify thoughts of suicide and refer them to the clinic. The therapists will work under the supervision of experts to support the participants.

\section{The Strengths of the Study}

A control group who will be treated with usual therapies will be included in the study. Effects of the intervention will be assessed in a long-term follow-up. Due to a design using comparative strategy to directly compare three intervention groups, this clinical trial will have external validity and the findings of this study will directly be applied in practice. One of the most important strengths of external validity is the outcome assessment. Furthermore, this clinical trial enjoys internal validity because the therapists follow the treatment protocol, as well as calculating the inter-rater reliability of the primary outcome and employing reliable and valid instruments. Group therapy using a cognitive behavioral intervention is another advantage of this intervention. The group members are also satisfied since they help each other and interact during the group meetings in RCBT and CBT. This results in higher self-confidence, a sense of social competence, and a sense of belonging.

\section{Limitations of the Study}

Blinding is not possible for participants and therapists, though the statistician is blind. This will minimize the effect of information bias. Another limitation of the study is the amount of time devoted to individuals in the RCBT and CBT interventions. The unwillingness of the patients to present their problems in group discussions and their embarrassment is another clinical concern of the researcher.

\section{Funding}

This study was supported by the Research Deputy of Mazandaran University of Medical Sciences, Sari, Iran. 


\section{Acknowledgment}

This study is supported by a grant from the Research Council of Mazandaran University of Medical Sciences.

\section{Authors' Contributions}

The study design and assessments were conceptualized by $\mathrm{SHH}$. The first drafts of this paper produced by ZE, which SHH reviewed. ZE and NV developed substantial contributions to obtain of data and were involved in revising the manuscript also they wrote the parts of the manuscript related to the $\mathrm{CBT}$ and $\mathrm{RCBT}$ which reviewed by SHH, GHJB, MG, CBT also reviewed by AAZ. The researchers who are recruiting patients from Oncology center will be supervised by GHJ who is supervisor in Oncology Clinic. The manuscript related to statistical power and data analysis wrote and reviewed by JYC. The manuscript related to measure of anxiety and depressions biomarkers wrote by ZE and NV and reviewed by ARR. All authors read and approved the final manuscript.

\section{Competing Interests}

The authors declare that they have no competing interests.

\section{References}

1. Malvezzi M, Arfe A, Bertuccio P, Levi F, la Vecchia C, Negri E. European cancer mortality predictions for the year 2011. Annals of Oncology. 2011; 22(4):947-56.

2. Allemani C, Weir HK, Carreira H, Harewood R, Spika D, Wang XS, et al. Global surveillance of cancer survival 19952009: analysis of individual data for 25676887 patients from 279 population-based registries in 67 countries $(\mathrm{CON}$ CORD-2). Lancet. 2014 Nov 26.

3. O'Connor M, Christensen S, Jensen AB, Moller S, Zachariae R. How traumatic is breast cancer? Post-traumatic stress symptoms (PTSS) and risk factors for severe PTSS at 3 and 15 months after surgery in a nationwide cohort of Danish women treated for primary breast cancer. Br J Cancer. 2011 Feb 1; 104(3):419-26.

4. Galloway SK, Baker M, Giglio P, Chin S, Madan A, Malcolm $\mathrm{R}$, et al. Depression and anxiety symptoms relate to distinct components of pain experience among patients with breast cancer. Pain Research and Treatment.2012; 2012:851276.

5. Olin JJ. Cognitive function after systemic therapy for breast cancer. Oncology (Williston Park, NY). 2001; 15(5):613-8.

6. Meyers CA. How chemotherapy damages the central nervous system. J Biol. 2008; 7(4):11.
7. Shibayama O, Yoshiuchi K, Inagaki M, Matsuoka Y, Yoshikawa E, Sugawara Y, et al. Association between adjuvant regional radiotherapy and cognitive function in breast cancer patients treated with conservation therapy. Cancer Med. 2014 Jun; 3(3):702-9.

8. Montazeri A. Health-related quality of life in breast cancer patients: a bibliographic review of the literature from 1974 to 2007. Journal of Experimental andClinical Cancer Research. 2008; 27(1):32.

9. Faller H, Schuler M, Richard M, Heckl U, Weis J, Küffner R. Effects of psycho-oncologic interventions on emotional distress and quality of life in adult patients with cancer: systematic review and meta-analysis.J Clin Oncol. 2013; 31(6):787-93.

10. Blume J, Douglas SD, Evans DL. Immune suppression and immune activation in depression. Brain, Behavior, and Immunity. 2011; 25(2):221-9.

11. Raison CL, Miller AH. Depression in cancer: new developments regarding diagnosis and treatment. Biological Psychiatry. 2003; 54(3):283-94.

12. Raison CL, Capuron L, Miller AH. Cytokines sing the blues: inflammation and the pathogenesis of depression. Trends Immunol. 2006 Jan; 27(1):24-31.

13. Osborne L, Gispen F, Meilman S, Gocke A, Tosi D, Payne J. Increased inflammatory markers in late pregnancy depression: the role of progesterone and allopregnanolone. Brain, Behavior, and Immunity. 2015; 49:22-3.

14. Roque S, Correia-Neves M, Mesquita AR, Palha JA, Sousa N. Interleukin-10: a key cytokine in depression? Cardiovasc Psychiatry Neurol. 2009; 2009:187894.

15. Gazal M, Jansen K, Souza LD, Oses JP, Magalhães PV, Pinheiro R, et al. Association of interleukin-10 levels with age of onset and duration of illness in patients with major depressive disorder. Revista Brasileira de Psiquiatria. 2015; 37(4):296-302.

16. Dowlati Y, Herrmann N, Swardfager W, Liu H, Sham L, Reim EK, et al. A meta-analysis of cytokines in major depression. Biol Psychiatry. 2010 Mar 1; 67(5):446-57.

17. Tavakoli-Ardakani M, Mehrpooya M, Mehdizadeh M, Hajifathali A, Abdolahi A. Association between Interlukin-6 (IL-6), Interlukin-10 (IL-10) and depression in patients undergoing hematopoietic stem cell transplantation. International Journal of Hematology-Oncology and Stem Cell Research. 2015; 9(2):80.

18. Cipriani A, Santilli C, Furukawa TA, Signoretti A, Nakagawa A, McGuire H, et al. Escitalopram versus other antidepressive agents for depression. Cochrane Database Syst Rev. 2009;2.

19. Shiroma PR, Thuras P, Johns B, Lim KO. Emotion recognition processing as early predictor of response to 8 -week citalopram treatment in late-life depression. Int J Geriatr Psychiatry. 2014 Apr 4.

20. Lash TL, Pedersen L, Cronin-Fenton D, Ahern TP, Rosenberg CL, Lunetta KL, et al. Tamoxifen's protection against breast cancer recurrence is not reduced by concurrent use of the SSRI citalopram. Br J Cancer. 2008 Aug 19; 99(4):616-21. 
21. Rizzo M, Creed F, Goldberg D, Meader N, Pilling S. A systematic review of non-pharmacological treatments for depression in people with chronic physical health problems. J Psychosom Res. 2011 Jul; 71(1):18-27.

22. Garlick M, Wall K, Corwin D, Koopman C. Psycho-spiritual integrative therapy for women with primary breast cancer. J Clin Psychol Med Settings. 2011 Mar;18(1):78-90.

23. Fallah R, Golzari M, Dastani M, Akbari ME. Integrating spirituality into a group psychotherapy

24. program for women surviving from breast cancer. Iranian Journal of Cancer Prevention. 2011; 4(3):141-3.

25. Olver IN, Dutney A. A randomized, blinded study of the impact of intercessory prayer on spiritual well-being in patients with cancer. Altern Ther Health Med. 2012 Sep-Oct; 18(5):18-27.

26. Shaw B, Han JY, Kim E, Gustafson D, Hawkins R, Cleary J, et al. Effects of prayer and religious expression within computer support groups on women with breast cancer. Psycho-Oncology. 2007; 16(7):676-87.

27. Koenig HG. Religious versus conventional psychotherapy for major depression in patients with chronic medical illness: rationale, methods, and preliminary results. Depress Res Treat. 2012; 2012:460419.

28. Andrews JG, Oei TPS. The roles of depression and anxiety in the understanding and treatment of obstructive sleep apnea syndrome. Clinical Psychology Review. 2004; 24(8):1031-49.

29. McGregora BA, Antonib MH. Psychological intervention and health outcomes among women treated for breast cancer: a review of stress pathways and biological mediators. Brain, Behavior, and Immunity. 2009; 23(2):159-66.

30. Jafari N, Zamani A, Farajzadegan Z, Bahrami F, Emami H, Loghmani A. The effect of spiritual therapy for improving the quality of life of women with breast cancer: a randomized controlled trial. Psychology, Health andMedicine. 2013;18(1):56-69.

31. Paiva CE, Paiva BS, deCastro RA, Souza CP, de Paiva Maia YC, Ayres JA, et al. A pilot study addressing the impact of religious practice on quality of life of breast cancer patients during chemotherapy. J Relig Health. 2013 Mar; 52(1):18493.

32. Ursaru M, Crumpei I, Crumpei G. Quality of life and religious coping in women with breast cancer. Procedia - Social and Behavioral Sciences. 2014 Feb21; 114:322-6.

33. Pearce M, Koenig HG. Cognitive behavioural therapy for the treatment of depression in Christian patients with medical illness. Mental Health, Religion and Culture. 2013; 16(7):730-40.

34. Koenig HG. Religious versus conventional psychotherapy for major depression in patients with chronic medical illness: rationale, methods, and preliminary results. Depression Research and Treatment. 2012;2012.

35. Pearce MJ, Koenig HG, Robins CJ, Nelson B, Shaw SF, Cohen HJ, et al. Religiously integrated cognitive behavioral therapy: A new method of treatment for major depression in patients with chronic medical illness. Psychotherapy. 2015; 52(1):56.
36. Bayanzadeh S, Bolhary J, Dadfar M, Karimi Keisomi I. Effectiveness of cognitive-behavioral religious-cultural therapy in improvement of obsessive-compulsive patients. Razi Journal of Medical Sciences. 2005; 11(44):913-23.

37. Bellinger D, Berk L, Koenig HG, Daher D, Pearce M, Robins $\mathrm{C}$, et al. Religious involvement, inflammatory markers and stress hormones in major depression and chronic medical illness.Open Journal of Psychiatry. 2014; 4(4):335-52.

38. Walpole SC, McMillan D, House A, Cottrell D, Mir G. Interventions for treating depression in Muslim patients: a systematic review. J Affect Disord. 2013 Feb 15; 145(1):11-20.

39. Lim C, Sim K, Renjan V, Sam HF, Quah SL. Adapted cognitive-behavioral therapy for religious individuals with mental disorder: a systematic review. Asian J Psychiatr. 2014 Jun; 9:3-12.

40. Sharifi V, Asadi SM, Mohammadi MR, Homayoun KHA, Semnani Y, Shabanikia A, et al. Reliability and feasibility of the Persian version of the structured diagnostic interview for DSM-IV (SCID). Advances in Cognitive Science. 2004.

41. Montazeri A, Vahdaninia M, Ebrahimi M, Jarvandi S. The hospital anxiety and depression scale (HADS): translation and validation study of the Iranian version. Health and Quality of Life Outcomes. 2003; 1(1):14.

42. Koenig H. Cognitive behavioral treatments for depression in patients with chronic illness. Available from: wwwspiritualityand health dukeedu//Full\%20Protocol. 2014.

43. Sadeghi M, Ladari RB, Haghshenas M. A study of religious attitude and mental health in students of Mazandaran University of Medical Sciences. Journal of Mazandaran University of Medical Sciences. 2010; 20(75):70-5.

44. Aaronson NK, Ahmedzai S, Bergman B, Bullinger M, Cull A, Duez NJ, et al. The European organization for research and treatment of cancer QLQ-C30: a quality-of-life instrument for use in international clinical trials in oncology. Journal of the National Cancer Institute. 1993; 85(5):36576.

45. Hamid N, Behslideh K, Bayghi MA, Dehghanizadeh Z. A study of the effect of religion-based cognitive-behavior therapy on depression in divorced women. Journal of Family Counseling andPsychotherapy. 2011; 1(1):54-64.

46. Kontodimopoulos N, Ntinoulis K, Niakas D. Validity of the Greek EORTC QLQ-C30 and QLQ-BR23 for measuring health-related quality of life in breast cancer patients. Eur J Cancer Care (Engl). 2011 May; 20(3):354-61.

47. Montazeri A, Harirchi I, Vahdani M, Khaleghi F, Jarvandi S, Ebrahimi M, et al. The EORTC breast cancer-specific quality of life questionnaire (EORTC QLQ-BR23): translation and validation study of the Iranian version. Quality of Life Research. 2000; 9(2):177-84.

48. Sadeghi M, Ladari RB, Haghshenas M. A study of religious attitude and mental health in students of Mazandaran university of medical sciences. Journal of Mazandaran University of Medical Sciences. 2010; 20:(75):70-5.

49. Beck J. Cognitive behavior therapy: basics and beyond. 2nd ed. New York: Guilford Press; 2011. 